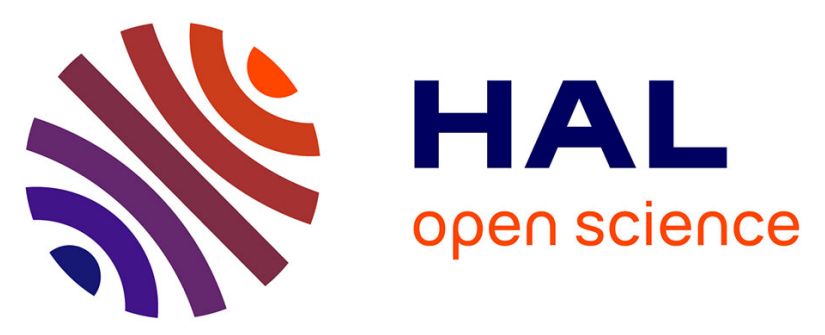

\title{
Light induced damage and repair in nucleic acids and proteins: general discussion Faraday Discussions
}

\author{
A. Chandra, Richard John Cogdell, Carlos E. Crespo-Hernandez, Ankona \\ Datta, Angelo Giussani, Stefan Haacke, John Helliwell, Roberto Improta, \\ Ramapurath Jayasree, Mike Jones, et al.
}

\section{To cite this version:}

A. Chandra, Richard John Cogdell, Carlos E. Crespo-Hernandez, Ankona Datta, Angelo Giussani, et al.. Light induced damage and repair in nucleic acids and proteins: general discussion Faraday Discussions. Faraday Discussions, 2018, 207, pp.389. 10.1039/C8FD90006J . cea-02328334

\section{HAL Id: cea-02328334 https://hal-cea.archives-ouvertes.fr/cea-02328334}

Submitted on 23 Oct 2019

HAL is a multi-disciplinary open access archive for the deposit and dissemination of scientific research documents, whether they are published or not. The documents may come from teaching and research institutions in France or abroad, or from public or private research centers.
L'archive ouverte pluridisciplinaire HAL, est destinée au dépôt et à la diffusion de documents scientifiques de niveau recherche, publiés ou non, émanant des établissements d'enseignement et de recherche français ou étrangers, des laboratoires publics ou privés. 


\title{
Light induced damage and repair in nucleic acids and proteins: general discussion
}

\author{
Amitava Chandra, Richard Cogdell, (D) Carlos E. Crespo-Hernández, (D) \\ Ankona Datta, Angelo Giussani, Stefan Haacke, (D) John Helliwell, (D) \\ Roberto Improta, Ramapurath S. Jayasree, Mike Jones, Tolga Karsili, \\ Bern Kohler, Mahil L, Imon Mandal, (D) Dimitra Markovitsi, (iD) \\ Himani Medhi, Padmaja P. Mishra, P. I. Pradeepkumar, Priyadarshi Roy \\ Chowdhury, Manas Sarangi, Igor Schapiro, (i) Ilme Schlichting, \\ Javier Segarra-Martí, (iD Amit Sharma, Vishnu V, Rienk van Grondelle \\ and Anthony Watts
}

\section{DOI: $10.1039 / C 8 F D 90006 J$}

Bern Kohler opened discussion of the paper by Mike Jones: What about charge transfer as a possible explanation for the emission quenching you observe with the cadmium telluride quantum dots?

Mike Jones answered: So far the data we have are consistent with a FRET mechanism, but we cannot rule out the involvement of charge transfer states. Professor Rienk van Grondelle has suggested we use Stark spectroscopy to explore this possibility.

Anthony Watts asked: Do the quantum dots (QDs) cause any spectral shift in your engineered photoproteins, as seen with QD coupling to bacteriorhodopsin?

Mike Jones replied: No, we haven't seen any influence of these QDs on the absorbance spectra of the various wild type and mutant RCs and RCLH1 complexes we have attached to them.

Richard Cogdell said: We see the quenching of the QD, but do you see the arrival of the energy at the reaction centre?

Mike Jones replied: Yes. We see enhanced P photobleaching in the presence of QDs and we see enhanced emission from a mutant RC that is engineered to lack the primary electron donor.

Anthony Watts asked: How do you know you've got that asymmetrical arrangement shown in your solar cell figure? Could controlling orientation be worth investigating? 
Mike Jones replied: In the case of electrode systems where the metal electrode is coated with a cytochrome $\mathrm{c}$ layer, an asymmetry of binding could be attributed to the cytochrome $\mathrm{c}$ binding site of the $\mathrm{RC}$ which is on the periplasmic side of the membrane - however the diagram is based on a rationalisation of the observed output and its likely mechanism rather than any independent evidence on the orientation of the protein at the electrode surface. There are certainly benefits to be had from controlling orientation and multiple studies have been carried out on this topic (mostly by other research groups in the field). We are currently working on strategies for engineering photoproteins for directed self assembly with electrodes and other materials, our preference being to obtain control through genetic coding (as in nature) rather than functionalisation of electrodes or complex fabrication processes.

Rienk van Grondelle asked: What is the cause of the difference between the original $8 \mu \mathrm{A} \mathrm{cm}^{-2}$ and the now $\sim 120 \mu \mathrm{A} \mathrm{cm}^{-2}$ photocurrent?

Mike Jones answered: The biggest influences on current are protein concentration and the choices of electrolyte, electrode materials and cell architecture.

Anthony Watts queried: What are the absolute currents that you generate, and if you want to take this into the field for an application, how large a surface would you need to produce power outputs necessary to drive a small device?

Mike Jones answered: We are some way from this at present because the high voltage devices we've been able to construct produce low currents and vice versa.

Stefan Haacke asked: Is this sketch real in the sense that you only have a monolayer of proteins on the electrode layer? In addition, what is the optimal thickness in terms of photocurrent density generated and what is the value you aim for?

Mike Jones responded: In some devices you can impose a monolayer on the electrode surface, or it is likely that this is what you achieve because proteins not adhered directly to the electrode are rinsed away during device fabrication (we can check loadings from the pigment content of the adhered proteins). In other devices we deliberately aim for multilayers of protein. It is difficult at this stage to say what an optimal thickness of protein might be, other than to say that what we are ultimately aiming for is a photocurrent output of the same order as that achieved in other types of solar cell (a few tens of $\mathrm{mA} \mathrm{cm}{ }^{-2}$ ).

Amit Sharma asked: How many photons are you putting into the system per protein molecule?

Mike Jones replied: We have not done that calculation.

Ilme Schlichting commented: Photosystem I has been used to photoreduce cytochrome P450, starting a catalytic reaction that is otherwise very difficult to trigger without using the natural component. Have you thought about using your system for catalysis applications? 
Mike Jones answered: Yes, this is something we are starting to explore alongside our work on photovoltaics.

John Helliwell communicated: Would crystal structure analysis of one or more of the authors' hybrids, such as depicted in Fig. $5 \mathrm{~b}$ in the paper, be worth a try to assist the authors' rational design towards optimisation of their nanodevices? Have they tried to crystallise one or more of their hybrids? ${ }^{1}$

1 As a guidebook see: N. E. Chayen, J. R. Helliwell and E. H. Snell, Macromolecular Crystallization and Crystal Perfection, International Union of Crystallography Monographs on Crystallography, Oxford University Press, 2010, ISBN: 9780199213252.

Mike Jones communicated in reply: We haven't attempted crystallisation of protein: QD conjugates but this is something we could consider, particularly for reaction centres where we have some experience of crystallisation.

Priyadarshi Roy Chowdhury communicated: What are the factors that are taken into consideration during the engineering of photoproteins?

Mike Jones communicated in response: Stability and expression level are really important for a mutation to be useful, so we need to avoid changes/additions to structure that interfere with assembly or destabilise the structure.

Priyadarshi Roy Chowdhury communicated: Why are PufX-deficient proteins incapable of conversion of solar energy in vivo?

Mike Jones communicated in reply: This is explained in our paper and summarised in Fig. 7. RCs in PufX minus RCLH1 complexes are unusually prone to "closing" under photosynthetic (i.e. reducing) growth conditions. By "closing" we mean the $\mathrm{Q}_{\mathrm{A}}$ quinone becomes reduced. This prevents charge separation forming a metastable radical pair state. Instead what happens is the antenna harvests light in the usual way, energy is passed to the $\mathrm{RC}$ in the usual way, the first two steps of charge separation happen in the usual way, but then because $\mathrm{Q}_{\mathrm{A}}$ is pre-reduced the $\mathrm{P}^{+} \mathrm{H}_{\mathrm{A}}^{-}$radical pair recombines (in nanoseconds) to the ground state and harvested energy is wasted as heat.

Dimitra Markovitsi opened discussion of the paper by Tolga Karsili: You mentioned that the 8-oxo-7,8-dihydro-2'-deoxyguanosine (8-oxoG) detected following near-UV irradiation of genomic DNA could stem from a photochemical path without involvement of the guanine radical cation. Do you expect the reaction intermediate identified in your calculations to react with spermine, which was shown to hinder 8 -oxoG formation ${ }^{1}$

1 M. Gomez-Mendoza, A. Banyasz, T. Douki, D. Markovitsi and J. L. Ravanat, J. Phys. Chem. Lett., 2016, 7, 3945-3948.

Tolga Karsili responded: Our model does not preclude a quenching of 8-oxoG when in the presence of spermine. Instead of reacting with water the photo-basic guanine will instead react with a more potent spermine molecule. In so doing, as with water, the initial step is likely to proceed via a photo-induced spermine-to- 
chromophore electron-driven proton-transfer reaction from $\mathrm{NH}$ of spermine to the N7-acceptor site of $\mathrm{G}$, followed by a subsequent self-reaction of the nascent $\mathrm{G}(+\mathrm{H})+$ spermine $(-\mathrm{H})$ radicals at the C8-site of $\mathrm{G}$.

Dimitra Markovitsi asked: Have you computed the absorption spectra of the reaction intermediates leading to 8-oxoG and pyrimidine hydrates? Such spectra could help identify these transient species by time-resolved spectroscopy, as was the case for the reaction intermediate leading to adenine dimerization. ${ }^{1}$

1 A. Banyasz, L. Martinez-Fernandez, T. Ketola, A. Muñoz-Losa, L. Esposito, D. Markovitsi and R. Improta, J. Phys. Chem. Lett., 2016, 7, 2020-2023.

Tolga Karsili replied: We are presently developing methods to compute detailed absorption spectra in bulk solution. This form of photodamage is an avenue we plan to apply our methods to in future. That said, your spectra are unlikely to reveal the transient intermediates that lead to the formation of the hydrate adducts since these are high energy intermediates that we predict to have lifetimes of the order of tens-of-fs. The possibility of computing the stable hydrate adducts themselves would however provide great insight into the formation mechanism for 8-oxoG.

Dimitra Markovitsi commented: According to your calculations the approach of a water molecule to the guanine is necessary for 8-oxoG formation. Do you expect this approach to be hindered in double strands or G-quadruplexes? Note that 8-oxoG was detected following irradiation of G-quadruplexes with a continuous light source at $266 \mathrm{~nm} .^{1}$

1 A. Banyasz, L. Martinez-Fernandez, C. Balty, M. Perron, T. Douki, R. Improta and D. Markovitsi, J. Am. Chem. Soc., 2017, 139, 10561-10568.

Tolga Karsili answered: Absolutely, the same photoreaction is unlikely to hold (at least to the same facile extent) in double-helix strands or G-quadruplexes. We note however that the formation of radical cations represents a chromophore-tosolvent charge-transfer rather than the mechanism outlined in our study which shows a solvent-to-chromophore charge-transfer. The nascent solvated-electron is likely to represent a very potent nucleophile which may abstract the $\mathrm{H}$-atom at the C8-site - thereby encouraging a reaction with water.

Anthony Watts said: Coming back to the question on absorption spectrum calculation, you mentioned in ref. 27 of your manuscript that some experimental data is available on this system - have you gone back and compared your data with experimental data?

Tolga Karsili replied: As highlighted in our manuscript, ref. 27 refers to a series of experiments performed on pyridine, seeded in gas phase water clusters. ${ }^{1}$ Using high-resolution spectroscopy, the work demonstrates the possibility for studying UV-induced water-to-chromophore intermolecular proton-transfer, in a gas-phase cluster, providing the possibility for testing our theoretical predictions for the type of proton-transfer that we presently predict as the initiator for the ensuing reaction path for forming lesion products. In order to draw synergies with the 
types of experiments performed by Prof. Markovitsi, we would need to compute the detailed absorption spectra of these hydrate intermediates in order to compare with the available data in the long-time resolved pump-probe experiments in the bulk. We are currently in the process of computing the details of such spectra at present.

1 N. Esteves-Lopez, S. Coussan, C. Dedonder-Lardeux and C. Jouvet, Phys. Chem. Chem. Phys., 2016, 18, 25637-25644.

Anthony Watts asked: If there was an ideal experiment to do to test out your model, what would it be?

Tolga Karsili responded: As I mentioned in my answer to your previous question, experiments to probe such water-to-chromophore proton-transfer studies are indeed available in the gas-phase ${ }^{1}$ for simple heterocyclic chromophores such as pyridine. The difficulty with the DNA/RNA nucleobases is their inherently low vapour pressures that preclude effective sample concentrations in gas phase. The gas-phase however provides a first-hand, detailed and high resolution probe for such photoreactions that are free from environmental perturbations. Analogous experiments to that of jet-cooled pyridine, seeded in water clusters, are possible with the DNA/RNA nucleobases and nucleosides (and associated oligomers) but would require experimental developments of methods that would increase the sample concentrations of a low vapour pressure system in the gas-phase. One such avenue would be the development of laser-desorption methods for desorbing DNA/RNA nucleobases that are bound to a rotating graphite disk. In solution, the ideal experiment that would test our present prediction would be time-resolved transient absorption spectroscopy. This would however require further theoretical modelling of the expected absorption profiles of the reaction intermediates in order to compare with the experimentally measured spectra.

1 N. Esteves-Lopez, S. Coussan, C. Dedonder-Lardeux and C. Jouvet, Phys. Chem. Chem. Phys., 2016, 18, 25637-25644.

Igor Schapiro asked: Are the reaction pathways that you have presented generated by interpolating geometries between the Frank Condon point, the conical intersection and the product geometry? If so, what type of conical intersection optimization have you done: minimum energy or minimal distance?

Tolga Karsili replied: In part. The potential energy (PE) profiles associated with the uracil/thymine + water photo-reactions were computed by means of a linear interpolation (in internal coordinates) between the Franck-Condon geometry and the geometry of a given minimum energy conical intersection. In the case of the thymine + water photo-reaction, in bulk double-helix DNA in aqueous solution, the ground and excited state PE profiles were computed by means of an excited state relaxed scan, using the $\mathrm{O}-\mathrm{H}$ stretch coordinate of the proximal water as the driving coordinate. Such relaxed scans were also used for computing the guanine + water photo-reaction in the gas phase. 
Bern Kohler noted: Photohydrate yields are reported to be greater for pyrimidine nucleotides than for the free bases. ${ }^{1}$ Can your model explain this observation? For example, could ribose substitution at N1 stabilize the carbocation?

$1 \mathrm{~J}$. Cadet and P. Vigny, The Photochemistry of Nucleic Acids, in Bioorganic Photochemistry, ed. H. Morrison, Wiley, New York, 1990, vol. 1, pp. 1-272.

Tolga Karsili answered: We would certainly expect the energetics of the various electronically excited states of the nucleotides to change relative to those of the isolated nucleobases. First however, we should clarify the nature of the reactive states as these do not lead to carbocations. In our model computations the charge-transfer (CT) state involves an electron promotion from a proximal water molecule to a $\pi^{*}$ orbital of the nucleobase. The CT state therefore contains a partial negative charge centered on the nucleobase. As with related solventchromophore systems, we predict that the CT state is populated by internal conversion from the initially excited locally-excited state which contains a nucleobase centered $\pi \pi^{*}$-character. The expected $\sigma$-donating nature of the ribose is therefore likely to destabilize the CT state but is likely to stabilize the final photohydrate via enhanced electron-density of the reduced $\pi$-system upon nucleophilic addition across C5-C6. This may lead to a greater yield of hydrate adducts in the nucleotides when compared to the nucleobases.

Javier Segarra-Martí asked: Have you compared your work to what has been done with $\mathrm{OH}$-substituted complexes? While the extra hydrogen you have with respect to $\mathrm{OH}$-substituted complexes will affect the decay paths, do you expect the absorption spectra to also change significantly?

Tolga Karsili replied: This is not a fair comparison. Hydroxyl-substituted uracil retains the same number of $\pi$-electrons as bare uracil but displays an understandable bathochromic shift in the absorption as a result of a $\pi$-donor substituent. C5-C6 hydrated uracil, in contrast, leads to a replacement of two of the six $\pi$-electrons of uracil into covalent bonding electrons - reducing the number of $\pi$ electrons to four. This inherent reduction of the $\pi$-electron density, upon going from uracil to 6-hydroxy-5H-uracil, is expected (and predicted in our manuscript) to vastly blue-shift the strong absorption bands in the hydrate adduct ( $c f$. uracil).

Anthony Watts commented: You said there are two parts to the PE profile generation to the hydrate, but you were talking earlier about radicals, where do radicals come in? Is there a difference between the gas phase and solution with respect to radical generation?

Tolga Karsili responded: The two step mechanism involves an initial electrondriven water-to-nucleobase proton-transfer and then a subsequent addition of the nascent $\mathrm{OH}$ radical to the odd-electron radical site of the nucleobase. The initial electronic excitation leads to the formation of local-excited (LE) $\pi \pi^{*}$ states - in which both the $\pi$ and $\pi^{*}$ orbitals are localized on the nucleobase. These LE states then couple to a near-lying charge-transfer (CT) state which involves an electron promotion from an oxygen $2 \mathrm{p}$ non-bonding orbital to the $\pi^{*}$ orbital of the nucleobase. This clearly represents a charge-separation in which the water 
contains a partial positive charge and the nucleobase contains a partial negative charge. The charge-separation is neutralized by water-to-chromophore proton $\left(\mathrm{H}^{+}\right)$transfer (i.e. the first step in the reaction process). This leaves a protonated chromophore $+\mathrm{OH}$ radical-pair intermediate - each of which contain an oddelectron from the initial excitation step. These nascent radicals will also persist in bulk aqueous solution since the formation of the nascent radical-pairs are dependent upon electronic excitation. The corresponding ion-pair states can only be formed by heterolytic bond fission of the ground state electronic configuration or an electronic excitation which involves an electron promotion from a $\pi$ orbital local to the chromophore and a $\pi^{*}$ orbital local to water. This will then follow hydrogen-atom (not proton) transfer from water to the chromophore to reveal the chromophore ${ }^{+}+\mathrm{OH}^{-}$ion-pair. Although these ion-pair states are stabilized in solution, our QM/MM computations on bulk DNA in aqueous solution reveal these states as higher energy states when compared to the water-to-chromophore CT states. This can be understood since DNA/RNA nucleobases contain enhanced basicities upon electronic excitation.

Mike Jones asked: How much of a problem is water for systems? I worry about water. The problem with protein technology is trying to keep the protein stable. Should we be working in an aqueous environment or trying to find a system that is less damaging such as a deep eutectic solvent?

Tolga Karsili responded: In certain environments, we and others have shown that photoreactions of water with solute molecules are common upon continuous light irradiation. Water is also problematic for time-resolved transient absorption experiments that exploit broadband IR wavelengths as probes, since the vibrational bands of water usually obstruct the important vibrational bands of the solute. As Prof. Bern Kohler showed, deep eutectic solvents offer an exciting alternative for overcoming such experimental problems but given their ionic nature, the extent to which they are photoreactive with a photoredox-active solute is unknown.

Himani Medhi communicated: The isolated gas phase of DNA/RNA units is free from environmental perturbations such as the water (cluster) and chromophore complex which are readily found in living systems. Thus, conventional photo-induction can damage DNA/RNA units in the gas phase more drastically than in bulk solution. Has this study found the same result as conventional ones or not?

Tolga Karsili communicated in reply: It is well-known that photohydrates are formed in DNA/RNA nucleobases in aqueous solutions, ${ }^{1}$ although their formation mechanisms are poorly understood. When comparing the gas phase potential energy profiles (Fig. 3 in our paper) with those returned in bulk aqueous doublehelix DNA (Fig. 8) it is evident that the excited-state nucleophilic hydrolysis reaction is in fact enhanced in the bulk. As Fig. 8 shows, the vertically excited bright S2 $\left(1 \pi \pi^{*}\right)$ state contains a far greater energetic driving force for coupling to the $\mathrm{S} 1(\mathrm{CT})$ state when compared to the restrictive barrier height in the gas phase (Fig. 3). This is understandable since charge-transfer states are known to stabilize upon dispersive $\pi-\pi$ interactions formed by intra-strand nucleobase stacking and inter-strand hydrogen-bonding. Our bulk DNA model features the nucleophilic 
hydrolysis reaction at the end-terminated nucleobase since we recognize that the interstitial regions of double helix DNA are water deficient. Such photoreactions with water are therefore unlikely to occur in chromosomal DNA but are likely to become important in short chain RNA and DNA strands as well as when bulk double-helix DNA unwinds for transcription.

$1 \mathrm{~J}$. Cadet and P. Vigny, The Photochemistry of Nucleic Acids, in Bioorganic Photochemistry, ed. H. Morrison, Wiley, New York, 1990, vol. 1, pp. 1-272.

Imon Mandal communicated: First, are 5 water molecules sufficient for the $\mathrm{G}+$ $\mathrm{H}_{2} \mathrm{O}$ system to describe the environment? How are the orientation of those waters going to affect the transitions and PES? Second, why is only one water taken for $\mathrm{T}$ and $\mathrm{U}$ but 5 waters are taken for the $\mathrm{G}$ system for the calculations?

Tolga Karsili communicated in reply: In order to describe the photo-reactive role of proximal water molecules, a quantum mechanical description of a subset of water molecules was required. Five is on the limit of the available resources that we can include with our choice of high-level multi-reference computational methods. As described in the manuscript, five water molecules are more than sufficient in describing all dominant first-order donor-acceptor effects as well as the secondary dispersive interactions. As outlined in the manuscript, our choice of five water molecules was successful in describing the chromophore-water interactions in related systems (both in our lab and by others - see ref. 21, 23 and 24 in our present manuscript). Since the orientation of the water molecules largely represent dispersive interactions and do not represent any first-order bonding interactions, the relative chromophore-water motions will show very minor changes in potential energy as a function of chromophorewater motions. So we conclude that small changes in the chromophore-water orientations will show very minor changes in the energetics associated with the reaction barriers, minima and conical intersections.

For the second question, this is a simple entropy argument - a single water molecule in the $\mathrm{G}+\mathrm{H}_{2} \mathrm{O}$ photoreaction would simply preclude a kinetically facile reaction since the dominant orbitals involved in the photoreaction are simply not optimally aligned. In contrast, the dispersively bound chromophore-water interaction in the $\mathrm{U} / \mathrm{T}+$ water photo-reaction leads to optimal alignment of the dominant orbitals that partake in the nucleophilic hydrolysis photoreaction.

Priyadarshi Roy Chowdhury communicated: What are the factors that lead to the photoinduced damage of DNA nucleobases?

Tolga Karsili communicated in reply: The obvious radiative factors are continuous exposure to near-UV radiation - such as those that lead to skin-cancer. Environmental factors are also important. Specifically, $\mathrm{pH}$ is an important factor since a reduced cellular $\mathrm{pH}$ is likely to enhance the probability of nucleophilic addition reactions to DNA. Other factors may include imbalances of ions/ molecules that provide optimal conditions within a cellular environment.

Tolga Karsili addressed Mike Jones: How does the photocurrent compare with that measured in industrial photovoltaics? 
Mike Jones responded: Dye sensitised solar cells typically produce an open circuit voltage of around $0.7 \mathrm{~V}$ and a short circuit current density of a few tens of microamps per $\mathrm{cm}^{2}$. The best photovoltages we have achieved are also around $0.7 \mathrm{~V}$, but the best photocurrents we have achieved are a little under 1 microamp per $\mathrm{cm}^{2}$. So we have some way to go as regards to current, and as I mentioned previously we are yet to produce both high currents and high voltages in the same device.

Mahil L asked: What led to the stability of the engineered protein vs. the natural protein?

Mike Jones replied: Our hypothesis is that closure of the LH1 ring increases stabilising protein-protein contacts between the central reaction centre and the inner ring of LH1 alpha-polypeptides, producing an overall stabilisation of the structure. Also the complete LH1 ring does not have any free ends, which may also assist in stabilisation. At this stage this is only a hypothesis. What is needed is a clearer picture of the process through which the native structure is lost, during thermal stress and also during photo-stress.

Richard Cogdell commented: You know there are thermophilic purple bacteria. Would it be possible to get further stabilisation by making your proteins more like the thermophilic ones? You could think of doing a more random approach as was done for GPCRs.

Mike Jones replied: Possibly. The random mutagenesis route is attractive but the challenge is always to have an effective screen.

Pradeepkumar P. I. asked Tolga Karsili: While showing the mechanism of addition of water to the C5-C6 bond of dC, you have shown a subsequent deamination reaction of $\mathrm{dC}$ to form $\mathrm{dU}$. Since deamination of $\mathrm{dC}$ is a common DNA damage which leads to GC to AU mutation, is there any chance that deamination happens prior to the addition of water to dC?

Tolga Karsili responded: We have not explored this subsequent reaction in our manuscript but it is an interesting query. Deamination is indeed common in DNA damage although such reactions are unlikely to proceed via initial $\mathrm{C}-\mathrm{NH}_{2}$ bond fission in the electronically excited state. This is because the only electronic state that forms a long-range dissociative interaction, with respect to $\mathrm{C}-\mathrm{N}$ bond elongation, is the ${ }^{3} \sigma \sigma^{*}$ state. Population of this state at the Franck-Condon region represents a spin-forbidden inter-system crossing from the initially excited singlet state. It is therefore more likely that the initial addition of water occurs across the C5-C6 bond of cytosine and then the nascent hydrate reacts with subsequent water molecules to eliminate $\mathrm{NH}_{2}$ - thus forming uracil hydrate + ammonia. This has already been shown experimentally by Boorstein et al. ${ }^{1}$

1 R. J. Boorstein, T. P. Hilbert, R. P. Cunningham and G. W. Teebor, Biochemistry, 1990, 29, 10455-10460.

Padmaja P. Mishra asked: Which nucleobases are more vulnerable to photodamage, DNA or RNA? 
Tolga Karsili answered: This is a rather general question, since there are several examples of photodamage of DNA/RNA nucleobases, involving different photoreactions. We have shown (and it has been experimentally confirmed) that uracil forms an enhanced yield for photo-induced nucleophilic addition of water across the C5-C6 of uracil. Prof. Markovitsi showed that adenine-thymine duplexes formed deprotonated radical-cation products and cycloadducts. Guanine is known to form an 8-oxoG lesion (as shown in our manuscript and others). It is well-known that stacked thymine dimers have an enhanced rate of $[2+2]$-cycloaddition. So, all nucleobases are vulnerable to photodamage, given optimal conditions and appropriate radiative environments.

Richard Cogdell opened a general discussion of the paper by Carlos E. CrespoHernández: If you think how DNA evolved, it evolved in deep ocean organisms that did not have to worry about UV damage. Do you think it was fortune that oceanic organisms evolved photostable DNA that let them colonise land, or did they need to evolve this over again once they got there?

Carlos E. Crespo-Hernández responded: Understanding the events that constitute the molecular origins of life is a major challenge in prebiotic chemistry. A period of chemical evolution may have preceded and shaped the progression from simple to complex molecules capable of performing biological functions. ${ }^{1,2}$ One hypothesis is that the chemistry that led to life began in the dark, or in highly UV-protected environments, such as in cracks and pores of solid surfaces, ${ }^{3}$ in clay minerals, ${ }^{4}$ beneath sacrificial molecular layers,${ }^{5}$ or below the surfaces of oceans. ${ }^{6-8}$ Although it is reasonable to hypothesize that a living organism capable of actively avoiding the surface of the ocean would not have to worry about UV damage, such a UV-shielding mechanism cannot protect prebiotic molecules that should have resurfaced periodically because of diffusion and mixing. ${ }^{9}$ As de Vries, Kohler, and co-workers eloquently proposed: "If life originated in fully UV-protected environments, UV selection pressure vanishes and less UV-hardy building blocks could just as easily have been precursors of the first self-replicating polymers. Moving prebiotic chemical evolution into the dark eliminates photochemical damage, but begs the question of how and when a transition to light-exposed environments took place. In contrast, starting life in the light is an attractive possibility that leads to UV-hardy molecules that avoid photodamage without losing the ability to support chemistry driven by longer wavelength photons." 9 Evidently, multiple selection pressures should have operated during early chemical and biological evolution as the driving force for the selection of the contemporary nucleobases. ${ }^{2}$ Extreme selection pressure for protection against intense UV radiation and photostability was likely a decisive criterion, ${ }^{6,10-12}$ which should have given some heterocycle analogues a selective advantage for their incorporation into the first informational polymers. ${ }^{2}$ Encoding such early polymers with highly photostable molecules is an elegant solution to the threat of photochemical damage. The intrinsic photostability to otherwise deleterious UV radiation observed in all five RNA and DNA nucleobases, provides strong support to the idea that UV radiation should have played a key role in the natural selection of the 'building blocks of life' during prebiotic Earth. 
1 W. Gilbert, Nature, 1986, 319, 618.

2 A. C. Rios and Y. Tor, Isr. J. Chem., 2013, 53, 469-483.

3 C. C. Bryce, G. Horneck, E. Rabbow and H. G. M. Edwards, Int. J. Astrobiol., 2015, 14, 115122.

4 J. D. Bernal, The physical basis of life, Routledge and Paul, London, 1951.

5 The Ecology of Cyanobacteria, ed. B. A. Whitton and M. Potts, Springer, 2000, pp. 591-611.

6 C. Sagan, J. Theor. Biol., 1973, 39, 195-200.

7 H. J. Cleaves and S. L. Miller, Proc. Natl. Acad. Sci. U. S. A., 1998, 95, 7260-7263.

8 M. J. Russell, A. J. Hall, J. Boyce and A. E. Fallick, Econ. Geol., 2005, 100, 419-438.

9 A. A. Beckstead, Y. Zhang, M. S. de Vries and B. Kohler, Phys. Chem. Chem. Phys., 2016, 18, 24228-24238.

10 C. E. Crespo-Hernández, B. Cohen, P. M. Hare and B. Kohler, Chem. Rev., 2004, 104, 19772019.

11 A. L. Sobolewski and W. Domcke, Europhys. News, 2006, 37, 20-23.

12 S. Ranjan and D. D. Sasselov, Astrobiology, 2016, 16, 68-88.

Ankona Datta asked: In your paper you mention that the reaction with prodrugs is going to create methylated products. What diseases are these targeted at? Do you want these molecules inserted into DNA?

Carlos E. Crespo-Hernández responded: The azathioprine, 6-mercaptopurine, and 6-thioguanine are effective anticancer, anti-inflammatory, and immunosuppressant drugs. ${ }^{1}$ These prodrugs undergo enzymatic conversion that ultimately culminates in the formation of 6-thioinosine and 6-thioguanosine nucleotides, which are required for their clinical effectiveness. As described in our article, the 6thioinosine nucleotide is in turn converted into the $S 6$-methylthioinosine nucleotide via the enzyme $S$-methyl transferase. ${ }^{2,3} S 6$-methylthioinosine can further inhibit purine de novo synthesis in vitro, ${ }^{4-6}$ and is linked to an increased risk of hepatotoxicity. ${ }^{7-9}$ The 6-thioguanosine nucleotide is metabolized into DNA, and the biological effects are partly dependent on the incorporation of 6-thioguanosine nucleotide into DNA. Once 6-thioguanosine is incorporated into DNA, it may undergo in situ non-enzymatic methylation to form S6-methylthioguanosine, which can induce lethal processing by DNA mismatch repair. ${ }^{10,11}$ As described in our article, methylation of 6-thioguanosine in DNA is largely responsible for the cytotoxic effects observed from these prodrugs in the absence of ultraviolet light, which have been correlated with an increased risk in leukemia ${ }^{11}$ and enhanced mutation frequency in circulating lymphocytes. ${ }^{12}$ Hence, methylation of 6-thioinosine and 6thioguanosine to form S6-methylthioinosine and S6-methylthioguanosine are naturally occurring events, which are at least partially responsible for the cytotoxic side effects associated with this treatment. One of the primary objectives of our study was to investigate the photochemical properties of S6-methylthioinosine in order to evaluate if it also has the potential to act as a phototoxic agent in the cell. Absorption of sunlight radiation by S6-methylthioinosine could lead to photochemical damage through light-induced reactions within the intracellular pool, as has been proposed for azathioprine and 6-thioguanine, ${ }^{13}$ and also suggested for other thionated DNA derivatives. ${ }^{14,15}$

1 P. Karran and N. Attard, Nat. Rev. Cancer, 2008, 8, 24-36.

2 K. G. Van Scoik, C. A. Johnson and W. R. Porter, Drug Metab. Rev., 1985, 16, 157-174.

3 R. Weinshilboum, Drug Metab. Dispos., 2001, 29, 601-605.

4 T. Dervieux, J. G. Blanco, E. Y. Krynetski, E. F. Vanin, M. F. Roussel and M. V. Rellin, Cancer Res., 2001, 61, 5810-5816.

5 T. Dervieux, T. L. Brenner, Y. Y. Hon, Y. Zhou, M. L. Hancock, J. T. Sandlund, G. K. Rivera, R. C. Ribeiro, J. M. Boyett, C.-H. Pui, M. V. Relling and W. E. Evans, Blood, 2002, 100, 1240-1247. 
6 S. A. Coulthard, L. A. Hogarth, M. Little, E. C. Matheson, C. P. F. Redfern, L. Minto and A. G. Hall, Mol. Pharmacol., 2002, 62, 102-109.

7 M. C. Dubinsky, S. Lamothe, H. Y. Yang, S. R. Targan, D. Sinnett, Y. Théorêt and E. G. Seidman, Gastroenterology, 2000, 118, 705-713.

8 M. C. Dubinsky, H. Yang, P. V. Hassard, E. G. Seidman, L. Y. Kam, M. T. Abreu, S. R. Targan and E. A. Vasiliauskas, Gastroenterology, 2002, 122, 904-915.

9 U. Nygaard, N. Toft and K. Schmiegelow, Clin. Pharmacol. Ther., 2004, 75, 274-281.

10 P. F. Swann, T. R. Waters, D. C. Moulton, Y.-Z. Xu, Q. Zheng, M. Edwards and R. Mace, Science, 1996, 273, 1109-1111.

11 J. Offman, G. Opelz, B. Doehler, D. Cummins, O. Halil, N. R. Banner, M. M. Burke, D. Sullivan, P. Macpherson and P. Karran, Blood, 2004, 104, 822-828.

12 T. Nguyen, P. M. Vacek, P. O’Neill, R. B. Colletti and B. A. Finette, Cancer Res., 2009, 69, 7004-7012.

13 M. S. Cooke, T. L. Duarte, D. Cooper, J. Chen, S. Nandagopal and M. D. Evans, DNA Repair, 2008, 7, 1982-1989.

14 M. Pollum, B. Ashwood, S. Jockusch, M. Lam and C. E. Crespo-Hernández, J. Am. Chem. Soc., 2016, 138, 11457-11460.

15 M. Pollum, L. Guan, S. Ahsanuddin, E. Baron, M. Lam and C. E. Crespo-Hernández, J. Invest. Dermatol., 2016, 136, S105.

Manas Sarangi asked: How is the conical intersection of the excited DNA base affected if you substitute the 2 and 6 position of the bases with methyl groups?

Carlos E. Crespo-Hernández answered: A concrete answer to this question requires multiconfigurational quantum-chemical calculations to be performed for those systems, which to my knowledge have yet to be done. We have shown that the fluorescence yield and the excited singlet state lifetime of O6-methylguanosine increase considerably compared to those of the guanine nucleoside or nucleotide (see our paper in this issue, DOI: 10.1039/c7fd00193b and Ashwood et al. ${ }^{1}$ ). Similarly, methylation of the amino group at the C6 position in adenine significantly changes the photophysical properties of N6,N6dimethyladenine compared to adenine. ${ }^{2-4}$ These photophysical changes suggest significant modifications to the order of the electronic states, to the topology of the excited-state potential energy surfaces, and/or to the access of key conical intersections upon methylation of the guanine and adenine monomers.

1 B. Ashwood, L. A. Ortiz-Rodríguez and C. E. Crespo-Hernández, J. Phys. Chem. Lett., 2017, 8, $4380-4385$.

2 B. Albinsson, J. Am. Chem. Soc., 1997, 119, 6369.

3 A. B. J. Parusel, W. Rettig and K. Rotkiewicz, J. Phys. Chem. A, 2002, 106, 2293-2299.

4 N. K. Schwalb and F. Temps, J. Phys. Chem. A, 2009, 113, 13113-13123.

Amitava Chandra said: I'm curious to know about possible applications of controlling the photochemistry by modifying the functional groups in nucleobases?

Carlos E. Crespo-Hernández responded: We have recently presented experimental and computational evidence showing that modification of the functional groups at the C2 and C6 positions of the purine nucleobases (or at the C2 and C4 position of the pyrimidines) regulates the rates of nonradiative and radiative decay. ${ }^{1}$ Modifications at C6 or C4 of the purine or pyrimidine chromophore, respectively, seems to play a particularly important role for a selected group of modified nucleobases. For instance, this knowledge can be used to identify prospective modified nucleobases for phototherapeutic ${ }^{2-4}$ and structural biology 
applications. ${ }^{5,6}$ It could also be used to predict the photoreactivity of a modified nucleobase, which can then be investigated to caution scientists about potential unintended consequences of modifying the genetic code. ${ }^{3}$

1 C. E. Crespo-Hernández, L. Martínez-Fernández, C. Rauer, C. Reichardt, S. Mai, M. Pollum, P. Marquetand, L. González and I. Corral, J. Am. Chem. Soc., 2015, 137, 4368-4381.

2 M. Pollum, S. Jockusch and C. E. Crespo-Hernández, J. Am. Chem. Soc., 2014, 136, 1793017933.

3 M. Pollum, B. Ashwood, S. Jockusch, M. Lam and C. E. Crespo-Hernández, J. Am. Chem. Soc., 2016, 138, 11457-11460.

4 M. Pollum, L. Guan, S. Ahsanuddin, E. Baron, M. Lam and C. E. Crespo-Hernández, J. Invest. Dermatol., 2016, 136, S105.

5 A. Favre, C. Saintomé, J.-L. Fourrey, P. Clivio and P. Laugâa, J. Photochem. Photobiol. B, 1998, 42, 109-124.

6 M. Pollum, S. Jockusch and C. E. Crespo-Hernández, Phys. Chem. Chem. Phys., 2015, 17, 27851-27861.

Ramapurath S. Jayasree asked: Have you looked into the singlet oxygen quantum yield? Is it comparable to or better than the FDA approved protoporphyrin IX or 5-ALA?

Carlos E. Crespo-Hernández replied: Yes, we have reported the yield of singlet oxygen generation for a large number of modified bases, ${ }^{1-6}$ with yields as high as $50 \%$. We have also shown that some of these modified bases are effective at diminishing the proliferation of skin cancer cells. ${ }^{4,7,12}$ Importantly, because these modified bases are often metabolized into the DNA of the skin cancer cells at a much faster rate than healthy cells, and they can be selectively excited by ultraviolet-A radiation, ${ }^{1,4,7,12}$ they do not necessarily require the presence of molecular oxygen to inflict damage to DNA, ${ }^{12}$ but can also damage DNA by direct photosensitized reactions. ${ }^{2,8-10}$ This is important because solid tumors are often deprived of molecular oxygen. ${ }^{11}$

1 M. Pollum, S. Jockusch and C. E. Crespo-Hernández, J. Am. Chem. Soc., 2014, 136, 1793017933.

2 M. Pollum, S. Jockusch and C. E. Crespo-Hernández, Phys. Chem. Chem. Phys., 2015, 17, 27851-27861.

3 M. Pollum, L. A. Ortiz-Rodríguez, S. Jockusch and C. E. Crespo-Hernández, Photochem. Photobiol., 2016, 92, 286-292.

4 M. Pollum, B. Ashwood, S. Jockusch, M. Lam and C. E. Crespo-Hernández, J. Am. Chem. Soc., 2016, 138, 11457-11460.

5 B. Ashwood, S. Jockusch and C. E. Crespo-Hernández, Molecules, 2017, 22, 379.

6 B. Ashwood, S. Jockusch and C. E. Crespo-Hernández, J. Phys. Chem. Lett., 2017, 8, 23872392.

7 M. Pollum, L. Guan, S. Ahsanuddin, E. Baron, M. Lam and C. E. Crespo-Hernández, J. Invest. Dermatol., 2016, 136, S105.

8 P. Clivio, J. L. Fourrey, J. Gasche and A. Favre, J. Am. Chem. Soc., 1991, 113, 5481-5483.

9 A. Favre, C. Saintomé, J.-L. Fourrey, P. Clivio and P. Laugâa, J. Photochem. Photobiol. B, 1998, 42, 109-124.

10 O. Reelfs, P. Macpherson, X. Ren, Y.-Z. Xu, P. Karran and A. R. Young, Nucleic Acids Res. 2011, 39, 9620-9632.

11 M. Höckel and P. Vaupel, J. Nat. Cancer Inst., 2001, 93, 266-276.

12 M. Pollum, L. Minh, S. Jockusch, C. E. Crespo-Hernández, ChemMedChem, 2018, DOI: 10.1002/cmdc. 201800148.

Vishnu V communicated: In the calculations performed, the solvent taken is acetonitrile. Won't the cellular environment affect the photochemical properties 
of the byproducts under study and what is (or can be) done to take into account such environmental effects?

Carlos E. Crespo-Hernández communicated in reply: The calculations reported in our article have been performed in vacuum, acetonitrile, and water environment for S6-methylthioinosine, and in vacuum and in acetonitrile for O6methylguanosine. Analogous calculations for 06-methylguanosine in a water environment have been reported recently in a separate contribution. ${ }^{1}$ The photochemistry of these byproducts in the cellular environment is currently unknown, but the experimental and computational results currently available (in DOI: 10.1039/c7fd00193b and in Ashwood et al. ${ }^{1}$ ) suggest that such investigations are necessary. Calculations that explicitly include the interaction of these byproducts with water molecules, amino acids, and/or nucleic acids could be performed at the quantum-mechanics/molecular-mechanics (QM/MM) level of theory, for instance, as have been widely discussed in this Faraday Discussions meeting for other systems.

1 B. Ashwood, L. A. Ortiz-Rodríguez and C. E. Crespo-Hernández, J. Phys. Chem. Lett., 2017, 8 , $4380-4385$.

Priyadarshi Roy Chowdhury communicated: How does the structural modification of the purine chromophore affect the electronic relaxation mechanisms?

Carlos E. Crespo-Hernández communicated in response: We have recently reported that structural modifications of the purine chromophore can significantly affect the electronic relaxation mechanism among other factors by altering the topology of the potential energy surfaces, their spin-orbit coupling interactions, and the access to key conical intersections. ${ }^{1,2}$ For instance, while excitation of the purine free base (i.e., the purine chromophore) efficiently populates a long-lived triplet state that decays in microseconds in aqueous solution, ${ }^{4}$ direct excitation of adenine (6-aminopurine) and guanine (2-amino6-oxopurine) monomers at $266 \mathrm{~nm}$ primarily results in the ultrafast decay (hundreds of femtoseconds) of the excited-state population by internal conversion to the ground state. Similarly, O6-methylation of the guanine monomers results in up to a 40 -fold increase in the excited singlet state lifetime of O6-methylguanosine compared to that of the guanine nucleoside or nucleotide (see DOI: 10.1039/c7fd00193b and Ashwood et al. ${ }^{3}$ ).

1 C. E. Crespo-Hernández, L. Martínez-Fernández, C. Rauer, C. Reichardt, S. Mai, M. Pollum, P. Marquetand, L. González and I. Corral, J. Am. Chem. Soc., 2015, 137, 4368-4381.

2 S. Mai, M. Pollum, L. Martínez-Fernández, N. Dunn, P. Marquetand, I. Corral, C. E. CrespoHernández and L. González, Nat. Commun., 2016, 7, 13077.

3 B. Ashwood, L. A. Ortiz-Rodríguez and C. E. Crespo-Hernández, J. Phys. Chem. Lett., 2017, 8, $4380-4385$.

4 E. Quiñones and R. Arce, J. Am. Chem. Soc., 1989, 111, 8218-8223.

Dimitra Markovitsi opened a discussion of the paper by Angelo Giussani: Is the mechanism you propose for formation of thymine-thymine (6-4) adducts consistent with the excitation wavelength dependence of their quantum yield determined experimentally? ${ }^{1}$ Experimental studies on thymine dimerization 
using triplet-triplet energy transfer detected only CPDs but not (6-4) adducts, see for example Sauvaigo et al. ${ }^{2}$

1 A. Banyasz, T. Douki, R. Improta, T. Gustavsson, D. Onidas, I. Vayá, M. Perron and D. Markovitsi, J. Am. Chem. Soc., 2012, 134, 14834-14845.

2 S. Sauvaigo, T. Douki, F. Odin, S. Caillat, J. L. Ravanat and J. Cadet, Photochem. Photobiol., 2001, 73, 230-237.

Angelo Giussani answered: Yes, the mechanism here proposed is consistent with the experimentally determined increase of the quantum yield of formation with the increase of the excitation energy, since also in the here proposed mechanism an energy barrier needs to be surmounted in order for the reaction to proceed. In the here proposed mechanism the barrier is placed on the gs PES. Thanks for the reference regarding thymine photoproducts using triplet-triplet energy transfer.

Igor Schapiro remarked: I want to ask about these two conical intersections that you have presented. They are so much below the Franck-Condon point that I think if you did dynamics simulations you would be able to overcome the barrier and access them. Is it feasible to run non adiabatic dynamics for this system?

Angelo Giussani answered: The two characterized conical intersections are indeed well below the vertical excitation energy of the CT state at the FC region, so the system excited in the CT should indeed be able to populate such degenerated regions. It must be noticed that the reported vertical excitation energy of the CT at the FC point is quite above the experimental excitation energy normally used and for which the 6-4 TT photodamage has been detected. Regarding possible dynamics simulations, I am aware that the group of Prof. Leticia Gonzalez has recently published dynamics simulations on the formation of the DNA photodamage cyclobutane pyrimidine dimer. ${ }^{1}$

1 C. Rauer, J. J. Nogueira, P. Marquetand and L. González, J. Am. Chem. Soc., 2016, 138, 15911-15916.

Carlos E. Crespo-Hernández commented: Interestingly, the participation of a triplet state with significant charge transfer character (i.e., a ${ }^{3} \mathrm{ct}-\pi \pi^{*}$-TT ${ }^{\prime}$ state, according to the nomenclature used in the manuscript) in the formation of the (64) photodimer is probably more likely in the thymine-thymine dinucleotide analogue thymidylyl $\left(3^{\prime}-5^{\prime}\right)$-4-thiothymidine, which has been shown to also form a (6-4) thietane photodimer upon selective irradiation of the 4-thiothymine moiety at $360 \mathrm{~nm} .^{1,2}$ This is so because recent transient absorption results for 4thiothymidine monomers ${ }^{3-5}$ have shown that intersystem crossing to the triplet manifold in this thymine analogue occurs in hundreds of femtoseconds and with nearly unity triplet yield.

1 P. Clivio, J. L. Fourrey, J. Gasche and A. Favre, J. Am. Chem. Soc., 1991, 113, 5481-5483.

2 P. Clivio, J.-L. Fourrey, J. Gasche and A. Favre, Tetrahedron Lett., 1992, 33, 1615-1618.

3 Y. Harada, C. Okabe, T. Kobayashi, T. Suzuki, T. Ichimura, N. Nishi and Y.-Z. Xu, J. Phys. Chem. Lett., 2010, 1, 480-484.

4 C. Reichardt and C. E. Crespo-Hernández, J. Phys. Chem. Lett., 2010, 1, 2239-2243.

5 M. Pollum, S. Jockusch and C. E. Crespo-Hernández, J. Am. Chem. Soc., 2014, 136, 1793017933. 
Tolga Karsili said: As a follow up to the comment by Prof. Crespo-Hernández, it is well-known that the efficient intersystem crossing displayed by simple carbonyl containing molecules (such as acetone) arises via the large changes in the $\mathrm{C}-\mathrm{O}$ bond distance of the lowest singlet and triplet state minima. This large difference in $\mathrm{C}-\mathrm{O}$ bond distance leads to a singlet/triplet crossing close to the minimum of the lowest singlet state, enhancing the spin-orbit coupling near the FranckCondon geometry. The near unity quantum yield for intercrossing crossing in the Paterno-Büchi reaction of simple carbonyls is a direct consequence of the aforementioned energetics. In the case of a $[2+2]$ cycloaddition of a thyminethymine dimer to form an oxetane, the intersystem crossing yield may be quenched (when compared to a simple Paterno-Büchi system) since the aromatic system will most likely restrict sufficient elongation of the $\mathrm{C}-\mathrm{O}$ bonds, manifesting in similar $\mathrm{C}-\mathrm{O}$ bond distances for the first/second singlet and triplet minima. Are the authors able to comment on whether their computations return significant changes in $\mathrm{C}-\mathrm{O}$ bond distance when comparing the singlet and triplet minima of common electronic state configurations?

Angelo Giussani responded: For the system here under study (i.e. a thymine dimer) the low-lying singlet and triplet states at the selected Franck-Condon geometry have $\pi \pi^{*}$ and $\mathrm{n} \pi^{*}$ nature. The $\pi \pi^{*}$ states (states $\mathrm{S} 1, \mathrm{~S} 2, \mathrm{~T} 1$, and $\mathrm{T} 2$, see Table 1 in our article) are mainly localized on the two C5-C6 carbon-carbon double bonds of the two thymine molecules, while the $\mathrm{n} \pi$ * states (states S3, S4, T3 and $\mathrm{T} 4$, see Table 1), are mainly localized on the two $\mathrm{C}_{4^{\prime}} \mathrm{O}$ carbonyl groups of the two thymine molecules.

For the studied system we have optimized the S1 $\pi \pi^{*}$ state, and the corresponding minima (see Fig. S7† in our article) display a slight elongation of the C5-C6 bond and a non-negligible elongation of the nearby carbonyl group $(+0.036$ $\AA)$. However this was the result of an optimization with a reduced active space (cas4,4) and in contrast with what was expected, that was a much pronounced elongation of the C5-C6 bond. The optimization of the singlet $n \pi^{*} \mathrm{~S} 4$ and triplet $\mathrm{n} \pi^{*} \mathrm{~T} 4$ states have resulted in minima in which the $\mathrm{C}_{4^{\prime}} \mathrm{O}$ bond displays a remarkable elongation, of plus 0.158 and $0.172 \AA$, respectively.

So we can conclude that for the present system, the optimization of the $\pi \pi^{*} \mathrm{~S} 1$, $\mathrm{S} 2$, T1, and T2 states probably leads to a slight elongation of the carbonyl group (we say probably because just the $\mathrm{S} 1$ state has been optimized here), while the optimization of the $\mathrm{n} \pi^{*} \mathrm{~S} 3, \mathrm{~S} 4, \mathrm{~T} 3$, and T4 leads to a much more substantial elongation of the $\mathrm{C}-\mathrm{O}$ bond. A similar degree of elongation is seen for the mentioned $n \pi^{*}$ minima, independently if singlet or triplet states (the $\mathrm{C}_{4^{\prime}} \mathrm{O}$ bond is equal to 1.370 and $1.384 \AA$ for the $1 \mathrm{n} \pi^{*}$ and $3 \mathrm{n} \pi^{*}$ minimum, respectively, see Fig. S7 $\dagger$ ), and again a similar degree of elongation is presumably forecast for the mentioned $\pi \pi^{*}$ states, independently if singlet or triplet states.

In comparing thymine with smaller systems such as acetone, we think that it is important to notice that while for acetone all excited states will be localized on the carbonyl group, in thymine the lowest excited states are instead mainly localized on the C5-C6 carbon-carbon double bond, which is of course not present in acetone. Probably an important difference in the Paterno-Büchi reaction between smaller molecules (as acetone and ethylene) and two thymine molecules, could in fact be caused by the fact that in thymine the main absorbing state is a $\pi \pi^{*}$ state 
on the C5-C6 bond, while in acetone the main absorbing state is already localized on the reactive carbonyl group.

We give thanks for this useful comment.

Roberto Improta asked: What happens when optimizing the geometry of the two lowest energy bright excited state states? What is their nature? Do you observe a significant mixing with CT states?

Angelo Giussani replied: For the present study we have optimized just one localized $\pi \pi^{*}$ state, the $1 \pi \pi^{*}$ - $\mathrm{T}^{\prime}$ state, this being the S1 state at the selected Franck-Condon geometry. As highlighted in our article, the performed optimization gave convergence problems, and for that reason it was performed with the reduced active space cas4,4 instead of the otherwise used cas8,6 active space. Along the performed optimization of the $1 \pi \pi^{*}-T^{\prime}$ state, the state preserves its nature, although a mixing with the $1 \mathrm{n} \pi^{*}-\mathrm{T}^{\prime}$ state is observable, which is probably causing the final structure to present geometrical modifications similar to the ones observed along the optimization of the $1 \mathrm{n} \pi^{*}-\mathrm{T}^{\prime}$ state. Along the performed optimization of the $1 \pi \pi^{*}-\mathrm{T}^{\prime}$ state a significant mixing with CT states was not observed.

Dimitra Markovitsi opened a general discussion of the papers by Carlos E. Crespo-Hernández and Angelo Giussani: Could you comment on how noncanonical bases may damage DNA?

Carlos E. Crespo-Hernández replied: This is obviously a broad and important question, but I will simply provide a generic answer herein. Non-canonical bases can potentially induce damage to DNA by both direct and indirect photosensitized reaction pathways. The specific nature of the damage will depend, among other factors (such as change in the DNA secondary or tertiary structure, base pairing and stacking interactions, etc.), on the electronic structure and the photophysical and physicochemical properties (such as redox potentials, fluorescence and triplet yields, etc.) of the precise non-canonical base. This is in part the reason for the necessity of investigating the photophysical and photodynamic properties of biologically relevant modified nucleobases thoroughly, as was one of the primary objectives of our study with S6-methylthioinosine and O6-methylguanosine. ${ }^{1}$

1 B. Ashwood, L. A. Ortiz-Rodríguez and C. E. Crespo-Hernández, Faraday Discuss., 2018, DOI: 10.1039/c7fd00193b; B. Ashwood, L. A. Ortiz-Rodríguez and C. E. Crespo-Hernández, J. Phys. Chem. Lett., 2017, 8, 4380-4385.

Angelo Giussani remarked: Regarding the formation mechanism of the 6-4 Thy-Thy adduct, it is important to remember that the time scale of the photoactivated part of the reaction, leading to the oxetane intermediate, is still unknown, so we don't know if the reaction happens (or not) in an ultrafast fashion as with the cyclobutane pyrimidine dimer formation process.

Tolga Karsili said: I have a comment and a question. The comment is in regard to the previous question. At near-threshold excitation wavelengths, it is well- 
known that DNA/RNA nucleobases and related analogues decay by internal conversion via conical intersections that contain out-of-plane ring deformation geometries, which in the purine nucleobases (and analogues) involved out-ofplane motions of either C6 or C2. Such conical intersections are either accessed directly or indirectly via non-adiabatic coupling - but are usually barrierless (or at best weakly barriered) in such biological systems. Motions along $\mathrm{N}-\mathrm{H}$ bond elongation coordinates do indeed contain conical intersections but these are manifestations of $\pi \sigma^{*}$ states. As Prof. Crespo-Hernández mentioned, $\pi \sigma^{*}$ states are energetically inaccessible at the Franck-Condon geometry. At such geometries, access to $\pi \sigma^{*}$ states (at the applicable wavelengths reported by CrespoHernández et al.) requires non-adiabatic $\mathrm{H}$-atom tunneling from the initially prepared bright state. Tunneling along the $\mathrm{N}-\mathrm{H}$ coordinate is however restricted in the nucleobases (and analogues) by a large potential barrier connecting the initially prepared bright state to the $\pi \sigma^{*}$ state.

My question is to Prof. Crespo-Hernández: In the oligonucleotides, in which out-of-plane ring deformations are quenched, what role do you expect substitution at the $\mathrm{C} 6$ and $\mathrm{C} 2$ positions to have on the relevant excited states of an oligonucleotide (e.g. charge-transfer states)?

Carlos E. Crespo-Hernández answered: I would like to first add to Dr Karsili's comment by directing readers to a recent work (and references therein) summarizing the evidence available regarding the participation of $\pi \sigma^{*}$ states in the excited-state dynamics of the nucleic acid bases. ${ }^{1}$

Concerning the consequences of substitution at the C6 and C2 positions of the purine chromophore on the excited state dynamics in oligonucleotides, the effect will depend on the specific modification done to the purine chromophore. The addition, removal, or substitution of a functional group from the C6 or C2 position of the purine chromophore in adenine or guanine is expected to modify the order of the electronic states and the topology of the excited-state potential energy surfaces of the modified base, ${ }^{2}$ and their redox properties. ${ }^{3}$ Together with the potential consequences of base stacking and base pairing interactions, photoexcitation of the modified base within a single- or double-stranded oligonucleotide can be expected to change the competition among different electronic relaxation pathways available in the oligonucleotide. A classic example is the simple substitution of adenine (6-aminopurine) by its structural isomer 2-aminopurine in oligonucleotides, which has been shown to significantly modify the excitedstate dynamics in DNA. ${ }^{4,5}$

1 G. M. Roberts and V. G. Stavros, Chem. Sci., 2014, 5, 1698-1722.

2 C. E. Crespo-Hernández, L. Martínez-Fernández, C. Rauer, C. Reichardt, S. Mai, M. Pollum, P. Marquetand, L. González and I. Corral, J. Am. Chem. Soc., 2015, 137, 4368-4381.

3 C. E. Crespo-Hernández, D. M. Close, L. Gorb and J. Leszczynski, J. Phys. Chem. B, 2007, 111, 5386-5395.

4 S. O. Kelley and J. K. Barton, Science, 1999, 283, 375-381.

5 C. Wan, T. Fiebig, O. Schiemann, J. K. Barton and A. H. Zewail, Proc. Natl. Acad. Sci. U. S. A., 2000, 97, 14052-14055.

Igor Schapiro said: Both papers have pointed out the importance of intersystem crossing. Is there a possibility either experimentally or computationally to use magnetic fields to explore this further? For example can you increase or 
decrease the intersystem crossing rate by applying a magnetic field in the nucleobases?

Carlos E. Crespo-Hernández replied: Early investigations have presented evidence that an applied magnetic field can modulate the fluorescence lifetimes and yields of pyrimidine in vacuum ${ }^{1-3}$ or the photoionization yield of 6-methylpurine in aqueous solutions. ${ }^{4}$ These experiments suggest mixing of singlet and triplet states with applied magnetic field for those two nucleobase derivatives. However, investigations focusing on the effect that an applied magnetic field has on the intersystem crossing rate of the canonical nucleobases have yet to be reported, perhaps because of their small triplet yields. Theoretical calculations are certainly possible.

1 N. Ohta, M. Fujita and H. Baba, Chem. Phys. Lett., 1987, 135, 330-334.

2 N. Ohta, T. Takemura, M. Fujita and H. Baba, J. Chem. Phys., 1988, 88, 4197-4203.

3 N. Ohta and T. Takemura, Chem. Phys., 1992, 162, 15-28.

4 C. Crespo-Hernández, R. Arce and E. Quiñones, Chem. Phys. Lett., 2003, 382, 661-664.

Dimitra Markovitsi asked: Could non-canonical bases behave as traps for the excitation energy in DNA as the minor natural base 5-methyl cytosine ${ }^{1}$

1 A. Banyasz, L. Esposito, T. Douki, M. Perron, R. Improta and D. Markovitsi, J. Phys. Chem. $B, 2016,120,4232-4242$.

Carlos E. Crespo-Hernández replied: In this article and in our previous investigation, ${ }^{1}$ we have shown that photoexcitation with ultraviolet B (UVB) radiation of both $S 6$-methylthioinosine and O6-methylguanosine can increase the probability of photochemical damage compared to the guanine nucleoside and nucleotide. However, it remains to be determined whether S6-/O6methylation of guanine chromophore increases DNA photodamage when these molecules are incorporated into DNA. We have shown that both S6methylthioinosine and O6-methylguanosine absorb radiation at lower energies than the guanosine nucleotide with larger absorptivity coefficients in the UVB spectral region, which increases the light penetration depth in cell tissue and thus probability of sunlight absorption. Furthermore, UVB excitation traps a significant fraction of the excited-state population in the excited singlet of 06 methylguanosine and in the triplet state of S6-methylthioinosine. For O6methylguanosine, a 40-fold increase in the excited singlet state lifetime compared to that of the guanine nucleoside or nucleotide is observed, which could increase the probability that 06 -methylguanosine may react with adjacent base-paired or base-stacked nucleobases when present in DNA. For S6methylthioinosine, the observation that a long-lived triplet state is populated upon UVB excitation suggests that photoexcitation of this molecule could potentially lead to oxidatively-generated cellular damage when present in the intracellular pool, as has been proposed for 6-thioguasonine and other thiopurine prodrugs. $^{2-5}$ Our study shows that further research is essential to investigate the possible photo-induced damage by these biological molecules to DNA and to other biological components within the cell.

1 B. Ashwood, L. A. Ortiz-Rodríguez and C. E. Crespo-Hernández, J. Phys. Chem. Lett., 2017, 8, 4380-4385. 
2 P. F. Swann, T. R. Waters, D. C. Moulton, Y.-Z. Xu, Q. Zheng, M. Edwards and R. Mace, Science, 1996, 273, 1109-1111.

3 P. O’Donovan, C. M. Perrett, X. Zhang, B. Montaner, Y.-Z. Xu, C. A. Harwood, J. M. McGregor, S. L. Walker, F. Hanaoka and P. Karran, Science, 2005, 309, 1871-1874.

4 Q. Gueranger, F. Li, M. Peacock, A. Larnicol-Fery, R. Brem, P. Macpherson, J. M. Egly and P. Karran, J. Invest. Dermatol., 2014, 134, 1408-1417.

5 R. Brem, M. Guven and P. Karran, Free Radical. Biol. Med., 2017, 107, 101-109.

\section{Conflicts of interest}

There are no conflicts to declare. 